\title{
Large squamous papilloma involving a transgender neovagina
}

\author{
Harold M Reed ${ }^{1 *}$, Joan C Delto ${ }^{2}$, Harry Sendzischew ${ }^{3}$, Pukhraz Basra ${ }^{4}$ and Lydia H Howard ${ }^{4}$ \\ ${ }^{1}$ The Reed Centre for Transgender Surgery, Bay Harbor Islands, Florida \\ ${ }^{2}$ Mount Sinai Medical Center Columbia University Division of Urology \\ ${ }^{3}$ Mount Sinai Medical Center Division of Surgery \\ ${ }^{4}$ Mount Sinai Medical Center Division of Pathology
}

\begin{abstract}
A 39-year-old Hispanic male to female transgender patient presents with a 6-month history of a protruding polypoid vaginal mass. She underwent vaginoplasty 11 years ago. Denies history of sexually transmitted diseases. MRI shows localized intravaginal mass. Excisional biopsy reveals squamous papilloma which stains negative for human papilloma virus (HPV). HPV acquisition is noted in $40 \%$ of sexually active college women within 2 years after initial contact. Routine HPV vaccination is advised for all female adolescents and transsexual vaginoplasty patients.
\end{abstract}

\section{Introduction}

Case: A 39-year-old Hispanic male to female transgender presented with a polypoid vaginal mass noted for 6 months. The patient has a past surgical history of breast implant placement at age 21 (revised at age 24), rhinoplasty at age 21 (revised age 36) and vaginoplasty at age 25. She is self-maintained on intramuscular Premarin 25 mg every 2 weeks. She has no known history of sexually transmitted diseases or anogenital skin lesions. An abdominal and pelvic MRI revealed hepatomegaly and a small right pre-sacral lymph node measuring $0.8 \mathrm{~cm}$. The rectum was unremarkable. Within the posterior wall of the neovagina a 1.9 $\mathrm{cm}$ lobulated and enhancing mass suggesting a possible malignancy was noted. Inguinal lymph nodes were not enlarged. Multiple injection granulomas were seen in the subcutaneous buttocks.

On physical examination, a mass was seen protruding from the introitus during Valsalva. Later that month, the tumor was completely removed by an excisional biopsy. The microscopic examination revealed a pedunculated mass composed of fibrovascular cores lined by squamous epithelium with hyperkeratosis, parakeratosis and focal acute inflammation. No epithelial atypia was identified. The histologic findings were consistent with a squamous papilloma. An immunohistochemical stain for p16, a surrogate marker for human papilloma virus (HPV) was negative. Androgen receptors are positive in the surrounding skin and adnexa, as well as within the papilloma. Stains for estrogen and progesterone receptors are negative. Gomori methenamine silver and Periodic acid Schiff stains for highlighting organisms were negative.

\section{Discussion}

Neooplastic growths of neovaginas are rare. Many of the reported neoplasms in neovaginas have been malignancies. Rare cases of benign neoplasms have been discussed [1]. The differential diagnosis of polypoid masses includes squamous papillomas, condyloma accuminata, and carcinoma [2].

Human papilloma virus infection is usually transmitted via sexual contact and can occur without penetration. Most HPV infections are transient and asymptomatic, causing no clinical manifestations.
Many studies have shown that greater than $90 \%$ of new HPV infections, including those with high-risk types, resolve or become undetectable within two years, and clearance usually occurs within the first 6 months after infection. In a prospective study of college women, the cumulative incidence of infection was $40 \%$ within 2 years after the first sexual contact [3]. HPV 16 accounted for only $10.4 \%$ of infections. Anogenital HPV is thought to be the most commonly transmitted infection in the United States. Over 14 million new HPV infections occur yearly involving an estimated 79 million actively infected, and half of this group are in the age range of $15-24$ years. $90 \%$

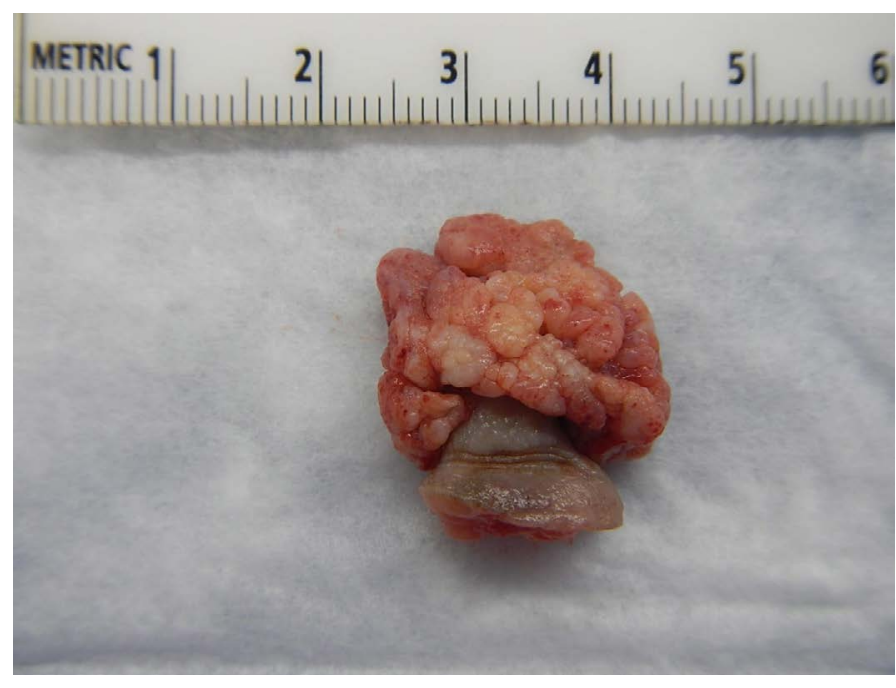

Figure 1. Gross A

Correspondence to: Harold M. Reed, MD, Diplomate of theAmerican Board of Urology, The Reed Centre for Transgender Surgery, Bay Harbor Islands, Florida,USA, Tel: 1-305-865-2000; E-mail: reed@srsMiami.com

Received: February 01, 2018; Accepted: February 16, 2018; Published: February 19,2018 


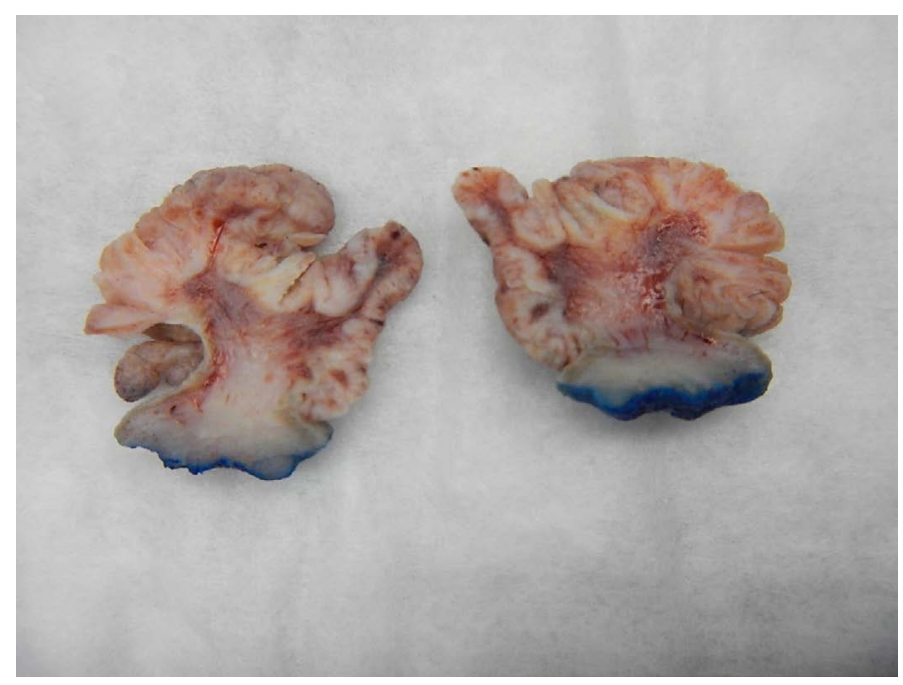

Figure 2. Gross B: Cut surfaces

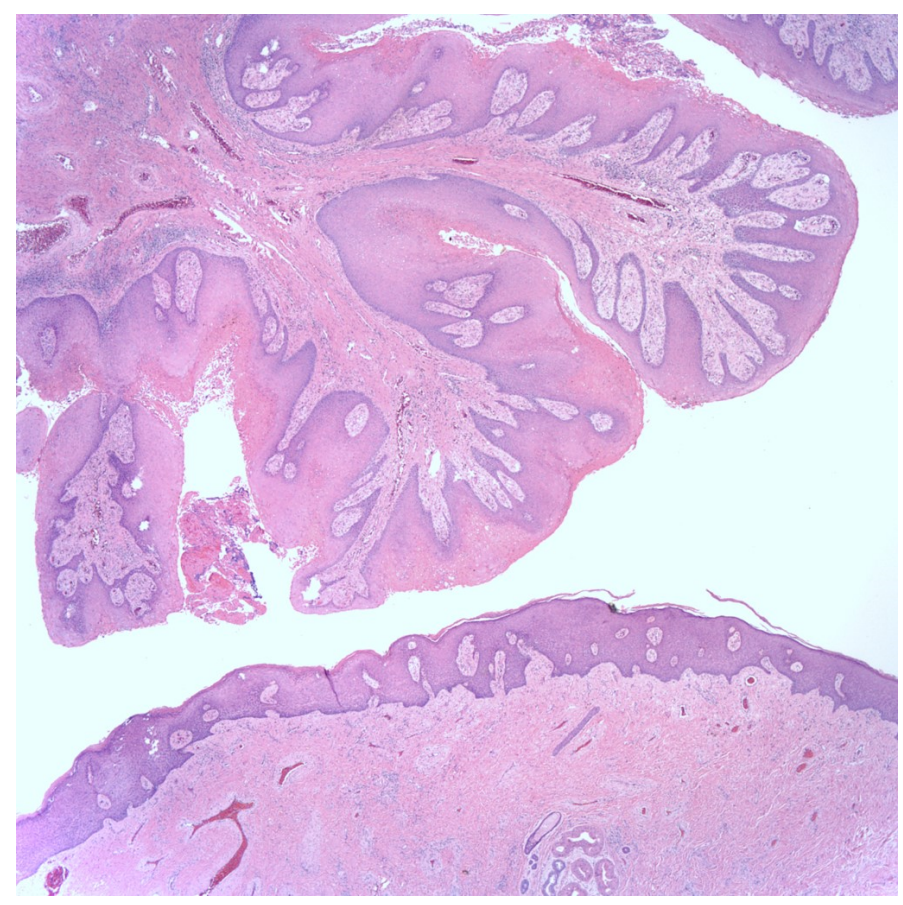

Figure 3. Micro: $\mathrm{H}$

of cases involved HPV virus serotypes 6 and 11. With HPV 6 and 11 the potential for malignant transformation is low. 118 papilloma viruses have been completely described and many more are suspected to exist by subgenomic aplicons [4]. They can cause warts as well as papillomas and may exist without being grossly visible [5].

The National Cancer Institute has recommended routine 3 dose HPV vaccination (type 9vHPV) at age 11 or 12 years [6]. For those not previously vaccinated or those who have not completed the 3 -dose course, females aged 13 through 26 years and of males aged 13 through 21 years may be vaccinated.

HPV vaccination and condom usage of is recommended for vaginoplasty patients. through age 26 years as well as men who have sex with men and for immunocompromised persons if not vaccinated previously. The quadrivalent (qHPV) vaccine Gardasil (Merck) also protects against HPV types 6 and 11 [7-9].
A p16 immunohistochemistry (IHC) is recommended by the American Society for Colposcopy and Cervical Pathology (ASCCP) when the H\&E morphologic differential diagnosis is between precancer and a mimic of precancer (such as atrophy and reparative epithelial changes); a strong and diffuse block-positive P16 result supports a categorization of precancerous disease." [2]. While the papilloma in our patient did not disclose atypia or suggestion of a precancerous lesion, a p16 IHC stain was performed as an academic adjunct.

Removal of the entire lesion and expert pathologic review is strongly advised before treatment is initiated [10].

\section{Conclusion}

HPV induced squamous papilloma occur frequently in the anogenital area. They are often invisible, are sexually transmitted, and typically benign. Their duration may be brief and self-limiting. Such lesions may grow to an unusually large size. Concurrent malignancy justifies excisional biopsy.

\section{Funding}

None

\section{References}

1. Braun H, Nash R, Tangpricha V (2017) Cancer in Transgender People: Evidence and Methodological Considerations. Epidermiol Review 39: 93-107 [Crossref]

2. Darragh TM, Colgan TJ, Cox JT, Heller DS, Henry MR, et al. (2013) The Lower Anogenital Squamous Terminology Standardization Project for HPV-Associated Lesions: background and consensus recommendations from the College of American Pathologists and the American Society for Colposcopy and Cervical Pathology. Int J Gynecol Pathol 32: 76-115 [Crossref]

3. Susan Hariri, Eileen Dunne, Mona Saraiya, Elizabeth Unger, Lauri Markowitz (2011) Manual for the Surveillance of Vaccine-Preventable Diseases, Centers for Disease Control and Prevention, Department of Health and Human Services, VPD Surveillance Manual, 5th Edition, 5 Human Papillomavirus: Chapter 5-1

4. DermNet New Zealand, Squamous Cell Papilloma, Ngan, Vanessa 2005

5. Rocky bacelieri (2005) Cutaneous warts: an evidence-based approach to therapy $\mathrm{Am}$ Fam Physician. 72: 647-652. [Crossref]

6. De Villiers EM, Fauquet C, Broker TR, Bernard HU, zur Hausen H (2004) Classification of papillomaviruses

7. Petrosky E, Bocchini JA, Hariri S (2015) Use of 9-Valent Human Papillomavirus (HPV) Vaccine: Updated HPV Vaccination Recommendations of the Advisory Committee on Immunization Practices. Morbidity and Mortality Weekly Report 64: 300-304. [Crossref]

8. Oelschlager AA, Kirby A, Breech L (2017) Evaluation and Management of Vaginoplasty Complications. Curr Opin Obstet Gynecol. 29: 316-321 [Crossref]

9. Chia-ching JW, Palefsky JM (2015) Human Papillomavirus (HPV) Infections and the Importance of HPV Vaccination Curr Epidemiol Rep 2: 101-109 [Crossref]

10. Indres MT, Deligdisch L, Altchek (2009) A Squamous Papilloma with Hyperpigmentation in the Skin Graft of the Neovagina in Rokitansky Syndrome. $J$ Pediatr Adolesc Gynecol 22: e148-e155 [Crossref]

Copyright: (C2018 Reed HM. This is an open-access article distributed under the terms of the Creative Commons Attribution License, which permits unrestricted use, distribution, and reproduction in any medium, provided the original author and source are credited. 